\title{
Percepción del uso de dióxido de cloro y grado de conocimiento sobre la COVID-19 en estudiantes de Medicina de una universidad peruana
}

\author{
Perception of the use of chlorine dioxide and degree of knowledge about COVID-19 in Medical students \\ from a Peruvian university
}

Percepção do uso de dióxido de cloro e grau de conhecimento sobre a COVID-19 em estudantes de Medicina de uma universidade peruana

Cesar Copaja-Corzo ${ }^{1,2 a}$

(iD https://orcid.org/0000-0002-3497-0158

Luis A. Céspedes-Hernández ${ }^{1,3 b}$

iD https://orcid.org/0000-0002-6660-7521

\section{Resumen}

Objetivo: Determinar la percepción del uso de dióxido de cloro (CDS) y el grado de conocimiento sobre la COVID-19 en estudiantes de Medicina de una universidad peruana. Material y métodos: Estudio observacional de corte transversal. Se realizó una encuesta virtual a 158 estudiantes de Medicina de la Universidad Nacional Jorge Basadre Grohmann, del 15 al 18 de agosto del 2020. El instrumento utilizado se dividió en tres secciones: características sociodemográficas, nivel de conocimientos sobre la COVID-19 y actitudes frente al uso del CDS. El análisis bivariado se realizó utilizando la prueba exacta de Fisher. Resultados: De los 158 estudiantes de Medicina encuestados, el 53 \% fueron mujeres, la edad media fue de 21 (RIC: 19-23) años. El $22 \%$ considera que si un médico o personal de salud recomienda el uso de dióxido de cloro, es porque tiene alguna utilidad para tratar la COVID-19, y el 39 \% tiene un conocimiento bajo sobre la enfermedad. Existe diferencia entre la percepción frente al uso de dióxido de cloro y el nivel de conocimientos sobre la COVID-19 $(p<0,05)$. Conclusión: Casi un cuarto de los estudiantes considera útil el uso de dióxido de cloro para tratar la COVID-19 si lo recomienda un médico o si la terapia médica convencional no surtiera efecto. Lo mencionado puede deberse a un nivel de conocimiento bajo sobre la enfermedad.

Palabras clave: COVID-19, pandemias, estudiantes de Medicina, educación médica, dióxido de cloro [Fuente: DeCS]

\section{Abstract}

Objective: To determine the perception of the use of chlorine dioxide (CDS) and the degree of knowledge about COVID-19 in medical students of a Peruvian university. Material and methods: Cross-sectional observational study. A virtual survey of 158 medical students from the Jorge Basadre Grohmann National University was conducted from 15 to 18 August 2020. The instrument used was divided into three sections: socio-demographic characteristics, level of knowledge about COVID-19 and attitudes towards the use of CDS. Bivariate analysis was performed using Fisher's exact test. Results: Of the 158 medical students surveyed, $53 \%$ were women, the average age was 21 (RIQ: 19-23) year-old. $22 \%$ consider that if a doctor or health care staff recommends the use

\footnotetext{
${ }^{1}$ Universidad Privada de Tacna. Tacna, Perú

${ }^{2}$ Centro de Investigación de Estudiantes de Medicina. Tacna, Perú

${ }^{3}$ Universidad Nacional Jorge Basadre Grohmann. Tacna, Perú

${ }^{a}$ Estudiante de Medicina Humana

${ }^{\mathrm{b}}$ Médico cirujano. Especialista en Neurología
} 
of chlorine dioxide, it is because it has some utility in treating COVID-19, and $39 \%$ have low knowledge about the disease. There is a difference between the perception regarding the use of chlorine dioxide and the level of knowledge about COVID-19 $(p<0.05)$. Conclusion: Almost a quarter of students consider the use of chlorine dioxide to treat COVID-19 useful if recommended by a doctor or if conventional medical therapy does not work. This may be due to a low level of knowledge about the disease.

Keywords: COVID-19, pandemics, medical students, medical education, chlorine dioxide [Source MeSH]

\section{Resumo}

Objetivo: Determinar a percepção do uso de dióxido de cloro (CDS) e o grau de conhecimento sobre a COVID-19 em estudantes de Medicina de uma universidade peruana. Material e métodos: Estudo observacional de corte transversal. Uma pesquisa virtual foi feita em 158 estudantes de Medicina da Universidade Nacional Jorge Basadre Grohmann, de 15 a 18 de agosto de 2020. O instrumento utilizado foi dividido em três seções: características sociodemográficas, nível de conhecimento sobre a COVID-19 e atitudes contra o uso do CDS. A análise bivariada foi feita com o teste exato de Fisher. Resultados: Dos 158 estudantes de Medicina entrevistados, $53 \%$ foram mulheres, a idade média foi de 21 (RIQ: 19-23) anos. $22 \%$ consideram que, se um médico ou pessoal de saúde recomenda o uso de dióxido de cloro, é porque tem alguma utilidade para tratar a COVID-19; $39 \%$ tem um conhecimento baixo sobre a doença. Existe uma diferença entre a percepção da utilização de dióxido de cloro e o nível de conhecimentos sobre a COVID-19 $(p<0,05)$. Conclusão: Quase um quarto dos estudantes considera útil o uso de dióxido de cloro para tratar a COVID-19 se recomendado por um médico ou se a terapia médica convencional não surtisse efeito. Isto pode ser devido a um baixo nível de conhecimento sobre a doença.

Palavras-chave: COVID-19, pandemias, estudantes de Medicina, educação médica, dióxido de cloro [Fonte: DeCS]

\section{Introducción}

La enfermedad del coronavirus del 2019 (COVID-19) fue declarada pandemia por la OMS, ${ }^{1}$ esta es causada por el virus SARSCoV-2, el cual selecciona las células huésped que expresan el receptor de la enzima convertidora de angiotensina-2 (AECA-2). ${ }^{2}$ La terapéutica utilizada no ha demostrado ser efectiva para prevenir el síndrome de hipersensibilidad. ${ }^{3}$ Uno de los tratamientos alternativos que no tienen sustento científico es el popular dióxido de cloro (CDS).

Los estudiantes de Medicina acceden en tiempo real a la información más actualizada, siendo importante determinar si tienen la capacidad de discernir e interpretar la evidencia científica.
Se procedió a realizar una encuesta donde se determinó el conocimiento acerca del dióxido de cloro, si era útil para prevenir e incluso "curar" la COVID-19, a pesar de conocer que en 2019 la Dirección General de Medicamentos, Insumos y Drogas (DIGEMID) ${ }^{4}$ alertó que dicho producto era deletéreo y que podría causar falla hepática y daños en el tracto gastrointestinal y respiratorio.

El CDS es un desinfectante que es utilizado para tratar aguas residuales, sin embargo, su difícil transporte y almacenamiento, hace que no sea muy utilizado en la actualidad, su punto a favor es su bajo costo de preparación y operación. ${ }^{5}$ Por otro lado, se hipotetizó que si el CDS podía neutralizar virus en superficies contaminadas, entonces podría ser utilizado por vía 
inhalatoria, dicha investigación nunca fue realizada por sus evidentes efectos adversos. ${ }^{6}$

En la actualidad los medios de comunicación, así como autoridades políticas en Perú, ${ }^{7}$ incluso muchos médicos, le han dado importancia a esta sustancia, indicando sus supuestos beneficios para la salud, aseverando que tiene un sustento científico, el mismo que es inexistente si se realiza la búsqueda bibliográfica.

El objetivo del estudio fue determinar la percepción del uso de CDS y el grado de conocimiento sobre la COVID-19 en estudiantes de Medicina de una universidad peruana.

\section{Material y métodos}

Se realizó un estudio observacional, retrospectivo y de corte transversal, en 158 estudiantes de Medicina Humana de la Universidad Nacional Jorge Basadre Grohmann en la ciudad de Tacna, Perú. La ejecución se dio en el periodo del 15 al 22 de agosto del 2020.

Se incluyó a estudiantes que contestaron la encuesta virtual de manera voluntaria, completa y de forma adecuada ( $\sin$ dejar ninguna respuesta vacía). Se excluyeron 25 encuestas que se encontraban incompletas (no tenían una o más de una respuesta) y 5 por no aceptar el consentimiento informado.

El muestreo fue no probabilístico, de tipo bola de nieve, donde cada encuestado invitó a participar a otro compañero de su promoción y estos a su vez compartían la encuesta a más estudiantes.

Para la medición del grado de conocimientos, actitudes sobre el uso de CDS y características sociodemográficas, se utilizó un cuestionario con 29 preguntas elaborado para esta investigación de la siguiente manera: primero se realizó una búsqueda bibliográfica de artículos científicos, al no existir instrumentos validados que se ajusten a la investigación decidimos elaborar uno; para la creación del instrumento se formó un grupo integrado por especialistas en medicina interna, neurología, educación médica y un estudiante de Medicina, quienes elaboraron el primer borrador del instrumento; luego de ello, se realizó un piloto con 10 alumnos de Medicina Humana a quienes se les preguntó por dificultades para entender el instrumento, con sus sugerencias se modificó el instrumento.

La encuesta se dividió en 3 secciones. La primera contiene características sociodemográficas: edad, género y año académico en el que se encontraban. La segunda parte incluye nivel de conocimientos de los estudiantes de Medicina sobre la COVID19; se evaluó utilizando 20 preguntas que constaban de información general sobre la COVID-19 (6 elementos), cuadro clínico (3 elementos), métodos diagnósticos (2 elementos), tratamiento (5 elementos) y prevención de contagio (4 elementos). A la respuesta correcta se le asignó 1 punto, a la incorrecta y "no tengo idea" se le asignó 0 puntos. Luego, se convirtió la puntuación total de nivel de conocimiento en percentil. Una puntuación de $\geq 75 \%$ se definió como un nivel de conocimiento "alto", una puntuación de 50$75 \%$ se definió como "moderado" y una puntuación de $\leq 50 \%$ se definió como "bajo". ${ }^{8}$ La tercera parte sobre actitud frente al uso de CDS propuso 6 afirmaciones que fueron evaluadas como de acuerdo, indiferente y en desacuerdo.

Luego de elaborar la encuesta y realizar el piloto, se aplicó la encuesta mediante el uso de la plataforma de Google Forms, que generó un enlace que fue enviado a los delegados de cada promoción de estudiantes, quienes a su vez enviaron el enlace de llenado a sus compañeros.

La investigación se adhirió a las normas de Helsinki, se respetó la privacidad de los datos brindados por los alumnos, garantizando el anonimato. Al inicio de la encuesta se colocó el consentimiento informado, el cual mencionaba la finalidad del trabajo, la publicación de los resultados globales y que en cualquier momento podían dejar de llenar la encuesta. Posteriormente, se generó una hoja de cálculo en el programa Microsoft Excel, en donde se realizó una primera limpieza de la base de datos y luego se pasó la información al programa estadístico Stata-MP v14, en donde se realizó un segundo control de calidad. 
Para el análisis descriptivo de las variables, se utilizó las frecuencias/porcentajes para las variables categóricas y la mediana/rango intercuartílico o media/desviación estándar para las variables numéricas (para ello se evaluó la normalidad de las variables, mediante la prueba estadística Shapiro Wilk).

Para conocer la asociación entre el nivel de conocimiento sobre la COVID-19 y la actitud sobre el uso de dióxido de cloro, se cruzaron las variables cualitativas utilizando la prueba estadística chi $^{2}$ o exacta de Fisher según sus valores esperados. Con ello, se obtuvo los valores $p$. Se consideró estadísticamente significativo cuando se obtuvo un valor $p$ menor de 0.05 .

\section{Resultados}

Un total de 158 participantes respondieron la encuesta. Se excluyeron 30 participantes (25 no completaron toda la encuesta y 5 no dieron su consentimiento informado). Finalmente, se incluyeron a 128 estudiantes de Medicina de la Universidad Nacional Jorge Basadre Grohmann que cursaban entre el primer y sexto año de estudios. La mediana de la edad fue 21 años (rango intercuartílico: 19-23 años); el 53,1 \% de participantes fueron mujeres; el $28,1 \%$ se encontraban en tercer año; el 45,3 y $39 \%$ de estudiantes tuvo un nivel de conocimientos moderado y bajo respectivamente sobre la COVID-19 (Tabla 1).

Al realizar el análisis bivariado, se encontró una relación estadísticamente significativa entre el nivel de conocimientos sobre la COVID-19 y la actitud: "En caso la terapia médica convencional para tratar la COVID-19 no surta efecto, se podría utilizar dióxido de cloro" $(p<0,05)$; "Si una persona se infecta por la COVID-19, se podría utilizar

\section{Tabla 1}

Características sociodemográficas y nivel de conocimientos sobre la COVID-19 en estudiantes de Medicina, Tacna 2020

\begin{tabular}{lc}
\hline Variable & $\mathrm{n}=128(\%)$ \\
\hline Edad & $21(19-23){ }^{*}$ \\
\hline Sexo & $68(53,13)$ \\
\hline Femenino & $60(46,88)$ \\
\hline Masculino & \\
\hline Año académico & $28(21,88)$ \\
\hline Primer año & $27(21,09)$ \\
Segundo año & $36(28,13)$ \\
Tercer año & $18(14,06)$ \\
Cuarto año & $7(5,47)$ \\
Quinto año & $12(9,38)$ \\
Sexto año & \\
\hline Nivel de conocimiento ** & $50(39,06)$ \\
\hline Bajo & $58(45,31)$ \\
Moderado & $20(15,63)$ \\
Alto & \\
* Mediana y rangos intercuartílicos \\
** Cálculo según percentil 0-50 = Bajo 50-75 = Moderado 75-100 = Alto
\end{tabular}


dióxido de cloro junto con la terapia médica convencional para aumentar la probabilidad de cura" ( $p<0,05)$; y "El dióxido de cloro puede prevenir el contagio por la COVID-19" $(p<0,05)$ (Tabla 2).
El $76 \%$ de los estudiantes de Medicina encuestados estuvo en desacuerdo con el uso de CDS como terapia para tratar la COVID-19, el $7 \%$ estuvo de acuerdo y al $17 \%$ le pareció indiferente. El $15 \%$ estuvo de acuerdo con

Tabla 2

Relación entre el nivel de conocimientos sobre la COVID-19 y la percepción frente al uso de CDS

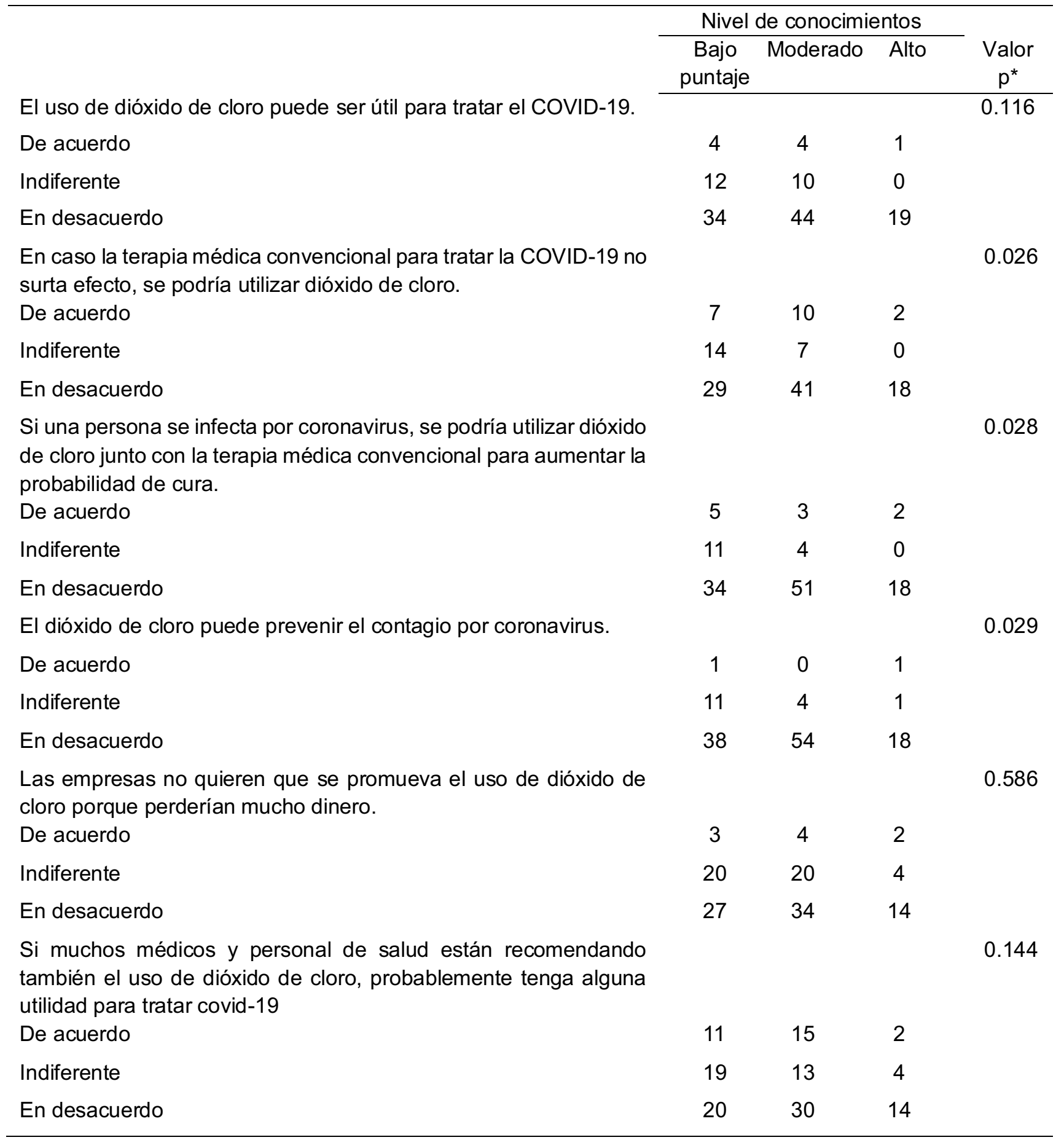

* Prueba estadística exacta de Fisher 
utilizar CDS como terapia para tratar la COVID19 si es que la terapia médica convencional no resultara efectiva, el $69 \%$ estuvo en desacuerdo y al $16 \%$ le pareció indiferente. El $80 \%$ estuvo en desacuerdo con utilizar el CDS junto con la terapia médica convencional para tratar la COVID-19, el $8 \%$ estuvo de acuerdo y al $12 \%$ le resultó indiferente.

La mayoría de los estudiantes (86 \%) estuvieron en desacuerdo con que el CDS puede prevenir el contagio por la COVID-19, el $2 \%$ estuvo de acuerdo y al $12 \%$ le fue indiferente. Al $34 \%$ de estudiantes le fue indiferente si el CDS hará perder dinero a las farmacéuticas, el $59 \%$ estuvo en desacuerdo y el $7 \%$, de acuerdo.

El $22 \%$ de los estudiantes de Medicina consideraron que si el personal de salud recomienda el uso de CDS es porque este sí tiene alguna utilidad para tratar la COVID-19, el $50 \%$ estuvo en desacuerdo y al $28 \%$ le pareció indiferente (Figura 1).

\section{Discusión}

El objetivo fue determinar la percepción del uso de CDS, el $22 \%$ de los estudiantes de Medicina cree que si un médico o personal de

\section{Figura 1}

Percepción de los estudiantes de Medicina acerca del uso de CDS, Tacna 2020

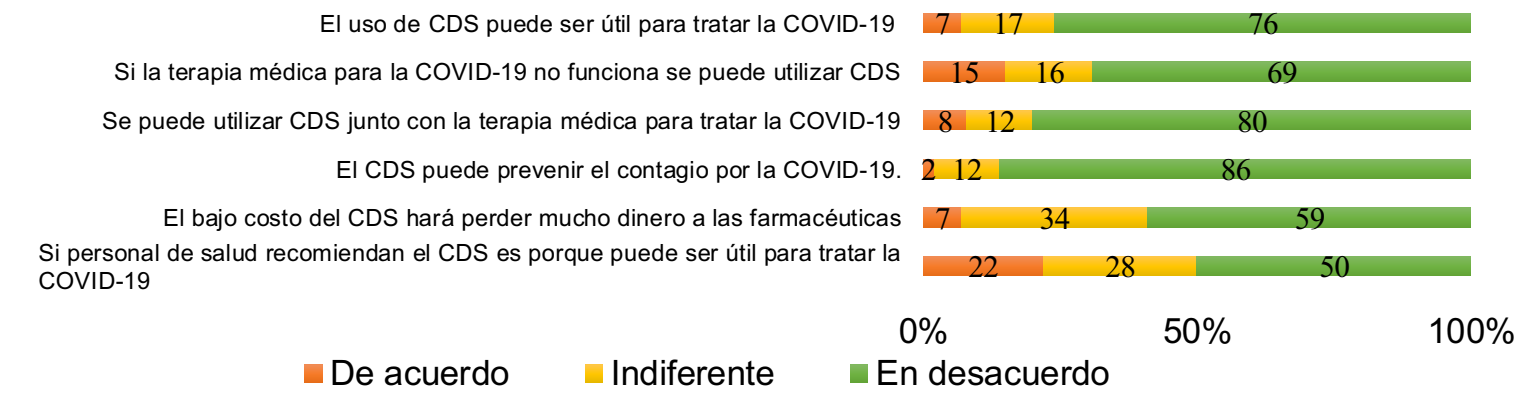

CDS: Dióxido de cloro; COVID-19: Enfermedad del coronavirus del 2019

salud recomienda su uso, es porque tiene alguna utilidad para tratar la COVID-19, esto coincide con el estudio de Azal Ikhlaq, quien determinó que de los estudiantes entrevistados, el $53 \%$ obtuvieron su conocimiento de la enfermedad de las redes sociales. ${ }^{9}$ En la actualidad, gran parte del conocimiento de la pandemia viene de medios digitales, siendo las opiniones de "expertos" las más difundidas, sin embargo, se debe tener en cuenta que es responsabilidad de los estudiantes discernir la mejor evidencia científica.

En relación con los conocimientos sobre la COVID-19, el estudio muestra que el 60,9\% de los estudiantes tiene un conocimiento entre alto y moderado $(15,6$ y $45,3 \%$ respectivamente), comparado con el estudio de
Francesca Gallè, quien observó que el $58 \%$ presentó un buen conocimiento del tema, ${ }^{10}$ ambos estudios coinciden en que los estudiantes de Medicina pueden responder preguntas complejas en relación con la forma de contagio, medidas preventivas, fisiopatología, clínica y tratamiento de la enfermedad.

Además, se determinó que el $39,1 \%$ de los estudiantes presentó un bajo nivel de conocimiento. Amin N. Olaimat determinó que solo un $3 \%$ de los estudiantes presentó un pobre nivel de conocimientos. ${ }^{11}$ Del mismo modo, el estudio de Francesca Gallè reveló que el $3,1 \%$ de los estudiantes presentó un bajo nivel de conocimiento. ${ }^{10}$ Esta diferencia de resultados es importante ya que permite estar al tanto de la brecha educacional que presentan 
los estudiantes, con relación a una enfermedad actual.

En el estudio, el $53,1 \%$ de los encuestados fueron de sexo femenino, además, el $71,1 \%$ de los estudiantes fueron de los primeros 3 años de estudios. Fatih Çalışkan et al. determinaron que el $55,3 \%$ de los encuestados fueron de sexo femenino, este estudio se realizó en estudiantes de último año, siendo un estudio que se realizó en 5 grandes universidades de Turkia. ${ }^{12}$ El presente estudio se ve limitado ya que solo se encuestó a estudiantes de una universidad de la localidad y la mayoría de los primeros años de estudio.

Pranav D. Modi, entrevistó a un $33 \%$ de estudiantes de Medicina, mientras que el resto de los estudiantes eran de otras ramas de las ciencias médicas y no médicas. ${ }^{13}$ Lo cual es otra limitante ya que el estudio solo incluyó a estudiantes de Medicina y no se puede comparar los conocimientos de otras poblaciones de estudiantes.

En el estudio, al $17 \%$ de los estudiantes le es indiferente si el CDS trata o no la COVID19 , además, el $86 \%$ de los estudiantes está en contra de utilizar el CDS como preventivo de infección por COVID-19, estos datos se pueden comparar con el estudio de Taype-Rondan, quien determinó que el $22,5 \%$ de los médicos generales entrevistados consideró que la eficacia de la homeopatía estaba científicamente comprobada. ${ }^{14}$ Lo anterior puede deberse al uso indiscriminado de las redes sociales y su influencia en la toma de decisiones y a los vacíos en la educación médica, respecto a terapias médicas alternativas.

El estudio tuvo como limitaciones, que la población evaluada no es representativa de todos los estudiantes de Medicina del Perú. Otra limitación fue que solo se evaluaron a estudiantes de Medicina, siendo necesario conocer la percepción en una población variada.

Se concluye que existe una asociación entre la precepción del uso de CDS y el nivel de conocimientos sobre la COVID-19. EI CDS es una sustancia deletérea, que no tiene sustento científico; sin embargo, al evaluar un grupo de estudiantes de Medicina de una universidad pública del Perú, el $22 \%$ considera útil el uso de dióxido de cloro para tratar la COVID-19 si lo recomienda un médico o si la terapia médica convencional no surtiera efecto. Lo mencionado podría deberse a un nivel de conocimiento bajo sobre la enfermedad.

Se recomienda reforzar el conocimiento de la medicina basada en evidencias y de terapias alternativas en la población estudiantil desde los primeros años, en especial en la actual pandemia, y programar capacitaciones acerca de la COVID-19 en la Escuela de Medicina.

\section{Referencias}

1. World Health Organization. Coronavirus disease (COVID-19). Situation Report - 168 [Internet]. Ginebra: WHO; 2020 [citado 13 de agosto de 2020]. Disponible en: https://www. who.int/docs/defaultsource/coronaviruse/situationreports/20200706-covid-19-sitrep168.pdf?sfvrsn=7fed5c0b_2

2. Sanders JM, Monogue ML, Jodlowski TZ, Cutrell JB. Pharmacologic treatments for Coronavirus Disease 2019 (COVID-19): A review. JAMA. 2020; 323(18):1824-183. Doi: 10.1001/jama.2020.6019

3. Mehra MR, Desai SS, Kuy SR, Henry TD, et al. Cardiovascular Disease, Drug Therapy, and Mortality in Covid-19, N Engl J Med 2020 ; 382 : e 102 . D o i : 10.1056/NEJMoa2007621

4. Ministerio de Salud. Consumo de productos que contienen clorito de sodio y/o dióxido de sodio, dióxido de cloro, representan un riesgo grave para la salud. Alerta DIGEMID No 41- 2019. [Internet] Lima, 2020 [citado 30 de agosto de $\left.\begin{array}{llll}2 & 0 & 2 & 0\end{array}\right]$. D i s p o n i ble e $n$ : http://www.digemid.minsa.gob.pe/UpLoad /UpLoaded/PDF/Alertas/2019/ALERTA_4 1-19.pdf?fbclid=IwAR1 dfiU $7 \mathrm{HjIx}$ OuEHMpFvYg2ZFZalJOlysFerszCW4KTi A_u5aEfPmAZ3uU 
5. Wang J, Shen J, Ye D, Yan X, Zhang Y, Yang W, et al. Disinfection technology of hospital wastes and wastewater: Suggestions for disinfection strategy during coronavirus Disease 2019 (COVID-19) pandemic in China. Environmental Pollution. 2020; 262. 114665, Doi: 10.1016/j.envpol.2020.114665

6. Karnik-Henry MS. Acidified Sodium Chlorite Solution: A Potential Prophylaxis to Mitigate Impact of Multiple Exposures to COVID-19 in Frontline Health care Providers. Hospital Practice. 2020; 48: $165-168$ D o i : 10.1080/21548331.2020.1778908

7. Álvarez A. Comisión del Congreso escuchará "recomendaciones" del "gurú" del peligroso dióxido de cloro, 21 de julio del 2020, [Internet]. Lima: La República 2020 [Consultado 30 de agosto 2020]. D i s p o n i b I e e $\mathrm{n}$ : https://larepublica.pe/politica/2020/07/21/c ongreso-comision-de-salud-escuchararecomendaciones-de-andreas-ludwig-elguru-del-peligroso-dioxido-de-clorocoronavirus/

8. Taghrir MH, Borazjani R, Shiraly R. COVID19 and Iranian Medical Students; A Survey on Their Related-Knowledge, Preventive Behaviors and Risk Perception. Arch Iran M ed. $2020 ; 23$ (4): $249-254$. Doi:10.34172/aim.2020.06

9. Ikhlaq A, Hunniya BE, Riaz IB, ljaz F. Awareness and Attitude of Undergraduate medical students towards 2019-novel

\section{Correspondencia:}

csarcopaja@gmail.com corona virus. Pak J Med Sci. 2020; 36:1-5. Doi: 10.12669/pjms.36.COVID19-S4.2636

10. Gallè F, Sabella EA, Da Molin G, De Giglio $O$. Understanding Knowledge and Behaviors Related to CoViD-19 Epidemic in Italian Undergraduate Students: The EPICO Study, Int J Environ Res Public Health. 2020, 17(10):2-11. Doi: 10.3390/ijerph17103481

11. Olaimat AN, Aolymat I, Shahba HM. Knowledge and Information Sources About COVID-19 Among University Students in Jordan: A Cross-Sectional Study. Frontiers in Public Health. 2020; 8:254. Doi: 10.3389/fpubh.2020.00254.

12. Çalışkan F, Midik O, Baykan Z, Senol Y. The knowledge level and perceptions towards COVID-19 among Turkish final year medical students. Postgraduate Medicine. $2020 ; \quad 132: 764-772$. Doi:10.1080/00325481.2020.1795486

13. Modi PD, Nair G, Uppe A, Modi J, Tuppekar B. COVID-19 Awareness Among Healthcare Students and Professionals in Mumbai Metropolitan Region: A Questionnaire- Based Survey. Cureus. 2020 ; 12 ( 4) : e 7514 . D o i : 10.7759/cureus.7514

14. Taype-Rondan A, Miñan-Tapia A, AlarconRuiz CA, Zafra-Tanaka JH. Factores asociados a la percepción de la validez científica de la homeopatía en un grupo de médicos generales de Perú. Rev Fac Med; 2019 ; $68(2): 215-221$. D o i : $10.15446 /$ revfacmed.v68n2.72116 